\title{
Oscillatory variations in the $Q$ factors of high quality micropillar cavities
}

Reitzenstein, S.; Gregersen, Niels; Kistner, C.; Strauss, M.; Schneider, C.; Pan, L.; Nielsen, Torben Roland; Höfling, S.; Mørk, Jesper; Forchel, A.

Published in:

Applied Physics Letters

Link to article, DOI:

$10.1063 / 1.3081030$

Publication date:

2009

Document Version

Publisher's PDF, also known as Version of record

Link back to DTU Orbit

Citation (APA):

Reitzenstein, S., Gregersen, N., Kistner, C., Strauss, M., Schneider, C., Pan, L., Nielsen, T. R., Höfling, S., Mørk, J., \& Forchel, A. (2009). Oscillatory variations in the $Q$ factors of high quality micropillar cavities. Applied Physics Letters, 94(6), 061108. https://doi.org/10.1063/1.3081030

\section{General rights}

Copyright and moral rights for the publications made accessible in the public portal are retained by the authors and/or other copyright owners and it is a condition of accessing publications that users recognise and abide by the legal requirements associated with these rights.

- Users may download and print one copy of any publication from the public portal for the purpose of private study or research.

- You may not further distribute the material or use it for any profit-making activity or commercial gain

- You may freely distribute the URL identifying the publication in the public portal 


\title{
Oscillatory variations in the $\boldsymbol{Q}$ factors of high quality micropillar cavities
}

\author{
S. Reitzenstein, ${ }^{1, a)}$ N. Gregersen, ${ }^{2}$ C. Kistner, ${ }^{1}$ M. Strauss, ${ }^{1}$ C. Schneider, ${ }^{1}$ L. Pan, ${ }^{1}$ \\ T. R. Nielsen, ${ }^{2}$ S. Höfling, ${ }^{\uparrow}$ J. Mørk, ${ }^{2}$ and A. Forchel ${ }^{1}$ \\ ${ }^{1}$ Technische Physik, Physikalisches Institut, Universität Würzburg, Am Hubland, D-97074 Würzburg, \\ Germany \\ ${ }^{2}$ Department of Photonics Engineering, DTU Fotonik, Technical University of Denmark, Building 343, \\ DK-2800 Kongens Lyngby, Denmark
}

(Received 19 January 2009; accepted 22 January 2009; published online 10 February 2009)

\begin{abstract}
We report on the observation of oscillatory variations in the quality $(Q)$ factor of quantum dot-micropillar cavities based on planar Bragg reflectors. The oscillatory behavior in the $Q$ versus diameter dependence appears in the diameter range between 1.0 and $4.0 \mu \mathrm{m}$, has a characteristic period of a few hundred nanometers and increases in amplitude with increasing reflectivity of the planar microcavity structures. The experimental results are well reproduced by numerical calculations which support the interpretation that the $Q$ oscillations are caused by coupling of propagating Bloch modes of different orders at the mirror interfaces. () 2009 American Institute of Physics. [DOI: 10.1063/1.3081030]
\end{abstract}

Confining light in semiconductor microcavities is a key issue for performing cavity quantum electrodynamics (cQED) experiments in solid state. Three dimensional (3D) photon confinements can be realized by a number of geometries including micropillar cavities, photonic crystal cavities, or microdisks. ${ }^{1}$ In all cases it is crucial to provide high $Q$ factor, low mode volume microcavities in order to obtain pronounced cQED effects such as weak or strong coupling on the single particle level. While the light-matter coupling strength $g$ is inverse proportional to the mode volume $V_{\text {mode }}$, the photon confinement time $\tau_{\mathrm{ph}}$ and, therefore, also the interaction time are proportional to the cavity $Q$ factor. In the present work we focus on micropillar cavities for which the cavity figure of merit for the observation of strong coupling is given by $Q / d_{c}$, where $d_{c}$ denotes the pillar diameter. ${ }^{2}$ For this type of microcavity, it is clear that a detailed knowledge on the cavity $Q$ as a function of the pillar diameter is a critical point in the technological optimization of the structures.

Experimental studies on different types of micropillars revealed a general drop in the pillar $Q$ factor with decreasing diameter, a tendency which is attributed partly to enhanced sidewall scattering but also tp less efficient mode confinement in the small diameter limit. ${ }^{3-6}$ In addition, investigations on submicron-diameter micropillars have shown an oscillatory variation in the $Q$ factor in agreement with theoretical predictions. ${ }^{7-9}$ In this submicron-diameter regime the oscillations of the $Q$ factor are related to the existence of a propagating Bloch mode providing an efficient route of energy transfer between the dominant $H E$ and $E H$ modes of the microcavity. The oscillatory variation in $Q$ is expected to be strongly damped for low $Q$ micropillars with diameters exceeding $1 \mu \mathrm{m}$. $^{8}$

In this letter, we present a detailed study of the $Q$ factor of high quality AlAs/GaAs micropillar cavities with diameters between 1 and $4 \mu \mathrm{m}$. This diameter range is of particular interest because it includes pillar diameters with the optimum $Q / d_{c}$-ratio of state-of-the-art micropillars with $Q$

${ }^{\text {a)} E l e c t r o n i c ~ m a i l: ~ s t e p h a n . r e i t z e n s t e i n @ p h y s i k . u n i-w u e r z b u r g . d e . ~}$ exceeding $150.000 .^{6}$ In the following we will address experimentally and theoretically the influence of the distributed Bragg reflector (DBR) mirror layout on the $Q$ factor of micropillars with different diameters. For this, arrays of micropillars of different diameters were patterned using an ultrasmall stepwidth $\left(\Delta d_{c}=20 \mathrm{~nm}\right)$. The small stepwidth was chosen to perform an in depth study of the diameter dependent pillar $Q$ factor. Our investigations reveal oscillatory variations of the $Q$ factor in the intermediate diameter range, which is most pronounced for very high- $Q$ samples. This finding is well reproduced by full $3 \mathrm{D}$ vectorial simulations.

The experiments were performed on micropillar cavities based on planar microcavity samples grown by molecular beam epitaxy. In order to study the influence of the cavity layout on oscillatory variations in the pillar $Q$ factors, four samples were grown with 20/23 (sample A), 23/26 (sample B), 26/29 (sample C), and 29/33 (sample D) AlAs and GaAs mirror pairs in the upper/lower DBR. The cavity was illuminated by InGaAs quantum dots located in the center of one $\lambda$ GaAs cavity sandwiched between the upper and lower DBRs. Arrays of micropillars were patterned by means of high resolution electron beam lithography and electron cyclotron resonance plasma etching. Each sample (A-D) contains several nominally identical arrays of micropillars with diameters $d_{c}$ between 1.0 and $4.0 \mu \mathrm{m}$, where the pillar diameter was varied by a step size of $\Delta d_{c}=20 \mathrm{~nm}$. Details on the growth and fabrication of the micropillars can be found in Refs. 6 and 10. A cross sectional scanning electron microscope (SEM) image of a processed $2.2 \mu \mathrm{m}$ micropillar is shown in Fig. 1. The image was obtained after parts of the micropillar had been removed by means of ion beam milling. Partial oxidation of the AlAs layers as well as a glass $\left(\mathrm{SiO}_{x}\right)$ coating layer are clearly visible.

Using low temperature $(10 \mathrm{~K})$ microphotoluminescence spectroscopy with a spectral resolution of $16 \mu \mathrm{eV}$ (Ref. 6) we determined the dependence of the cavity's fundamental mode $Q$ factor as a function of $d_{c}$ for samples A-D, where $Q$ is defined as the ratio of the resonance wavelength $\lambda_{c}$ and the mode linewidth $\Delta \lambda_{c}$ (full width at half maximum) $\left(Q=\lambda_{c} / \Delta \lambda_{c}\right)$. Here $\lambda_{c}$ depends on the particular cavity layout and the pillar diameter and lies in the range of $920-960 \mathrm{~nm}$ 


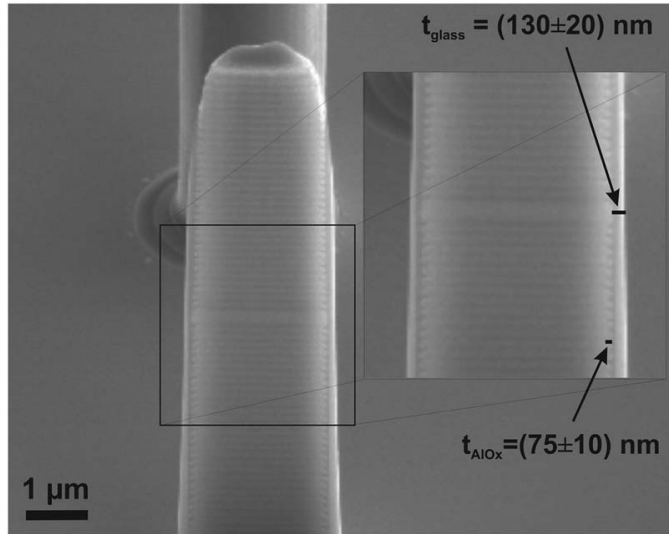

FIG. 1. Cross sectional SEM image of a micropillar cavity with a diameter of $2.2 \mu \mathrm{m}$ (measured at the site of the $\lambda$ cavity). The inset illustrates the appearance of the $\mathrm{AlO}_{x}$ oxide layer and the glass surface layer with a typical thickness of $t_{\mathrm{AlO}_{x}}=75 \mathrm{~nm}$ and $t_{\text {glass }}=130 \mathrm{~nm}$, respectively.

for the present samples. A typical $Q\left(d_{c}\right)$ dependence of sample C with 26/29 mirror pairs is presented in Fig. 2(a). The plot shows $Q$ factors for two independent arrays $(\mathrm{C} 1$ and $\mathrm{C} 2$ ) of micropillars as well as traces corresponding to a five point smoothing of the raw data. Two important features can be observed. First of all, the pillar $Q$ drops from about 50.000 to 5.000 when $d_{c}$ decreases from 4 to $1.2 \mu \mathrm{m}$, which is related to enhanced radiative and edge scattering losses in the small diameter limit. ${ }^{6}$ More importantly, the $Q\left(d_{c}\right)$ dependence shows strong oscillatory variations which have not been expected to appear in the diameter range under investigation. ${ }^{7,8}$ The oscillatory behavior that has not been observed before, mainly due to a rather rough scan of the $Q\left(d_{c}\right)$ dependence, has its largest amplitude at $d_{c} \approx 2.5 \mu \mathrm{m}$ and several nearby maxima can be observed [marked by black arrows in Fig. 2(a)]. The robustness of the effect is
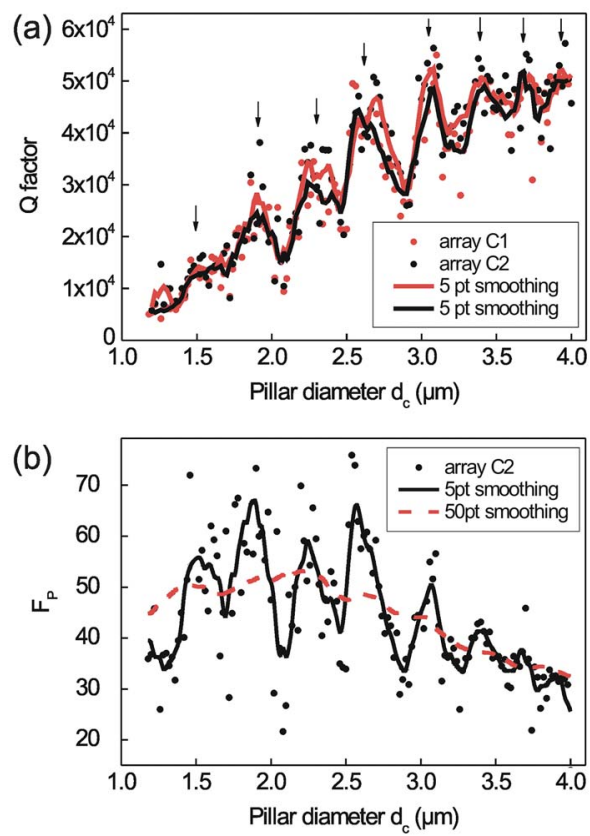

FIG. 2. (Color online) (a) Cavity $Q$ factors of sample C (26/29 mirror pairs in upper/lower DBR) as a function of pillar diameter. Almost identical oscillatory behavior is observed for two independent subarrays ( $\mathrm{C} 1$ and $\mathrm{C} 2)$ located at different positions on sample C. (b) Purcell factor $F_{P}$ calculated for array $\mathrm{C} 2$ vs pillar diameter. nicely reflected in the almost identical $Q\left(d_{c}\right)$ behavior measured for arrays $\mathrm{C} 1$ and $\mathrm{C} 2$ located at different positions on sample C.

While a detailed investigation of the oscillatory $Q$ behavior is interesting for the fundamental understanding of light confinement in micropillar cavities by its own right, it also has important implications on cQED experiments in this system. To demonstrate the latter we plotted the theoretical Purcell factor $F_{p}=3 Q \lambda_{c}^{3} / 4 \pi^{2} n^{3} V_{\text {mode }}$ based on the data in Fig. 2(a) (array C1) as a function of $d_{c}$ in Fig. 2(b). The oscillatory $Q$ variations are reflected in $F_{P}$ and result in strong deviations from the average $F_{P}$ values approximated by a 50 point smoothing of the raw data. Thus, while the average $F_{P}$ is almost constant at $d_{c} \approx 2 \mu \mathrm{m}\left(F_{P} \approx 50\right)$ the effective $F_{P}$ increases by almost a factor of 2 when decreasing $d_{c}$ from $2.1 \mu \mathrm{m}\left(F_{P} \approx 35\right)$ to $1.9 \mu \mathrm{m}\left(F_{P} \approx 65\right)$.

In order to model the experimental results and to gain a better understanding of the underlying phenomena we performed full 3D vectorial simulations for which we have employed the eigenmode expansion technique ${ }^{11}$ with improved perfectly matched layers. ${ }^{12}$ Following Ref. 9, we take into account two types of fabrication-induced imperfections, namely, the selective oxidation of AlAs from the sides and the appearance of a glass coating layer. The thickness $t_{\text {glass }}$ of the glass coating and the oxidation depth $t_{\mathrm{AlO}_{x}}$ are estimated by inspecting Fig. 1 (inset).

We compute the $Q$ factor by considering the cavity roundtrip operator described by the matrix $\mathbf{R}=\mathbf{R}_{\mathrm{T}} \mathbf{P} \mathbf{R}_{\mathrm{B}} \mathbf{P}$. Here $\mathbf{R}_{\mathbf{T}}$ and $\mathbf{R}_{\mathbf{B}}$ are the reflection matrices for the top and bottom DBRs, respectively, and $\mathbf{P}$ is a diagonal matrix describing propagation inside the cavity. When performing a full 3D computation of the $Q$ factor, the resonant cavity mode is a linear combination of cavity eigenmodes with coefficients given by the vector $\vec{c}$. These coefficients are determined by solving the eigenmode problem

$$
\mathbf{R} \vec{c}=R_{r} \vec{c},
$$

where $R_{r}$ is the eigenvalue describing the roundtrip of the resonant mode and the resonance wavelength $\lambda_{c}$ is determined by requiring $\arg \left(R_{r}\right)=0$. The $Q$ value is then given by ${ }^{13}$

$$
Q=\frac{-\lambda_{c}}{2\left(1-\left|R_{r}\right|\right)} \frac{\delta}{\delta \lambda} \arg \left(R_{r}\right) .
$$

In the linear combination $\vec{c}$, the fundamental mode is dominating; however, the importance of including higher order modes to understand oscillations in $Q$ was demonstrated in Refs. 7 and 8.

To investigate the origin of oscillations in the longdiameter regime, we examine experimentally and numerically the influence of the number of mirror pairs in the upper and lower DBRs on the pillar $Q$ value. Experimentally, we observe a pronounced influence of the DBR layout on the $Q\left(d_{c}\right)$ dependence, which is demonstrated in Fig. 3(a). It is seen that the higher reflectivity of DBRs with larger number of mirror pairs is reflected in larger overall $Q$ factors. In addition, the $Q$ oscillations are significantly stronger for high $Q$ samples $\mathrm{C}$ and D compared to intermediate $Q$ and low $Q$ samples B and A, respectively. The corresponding theoretical results are presented in Fig. 3(b). In agreement with the experiment, oscillations are nearly absent for 20/23 layer pairs in this diameter regime. When increasing the number of pairs 

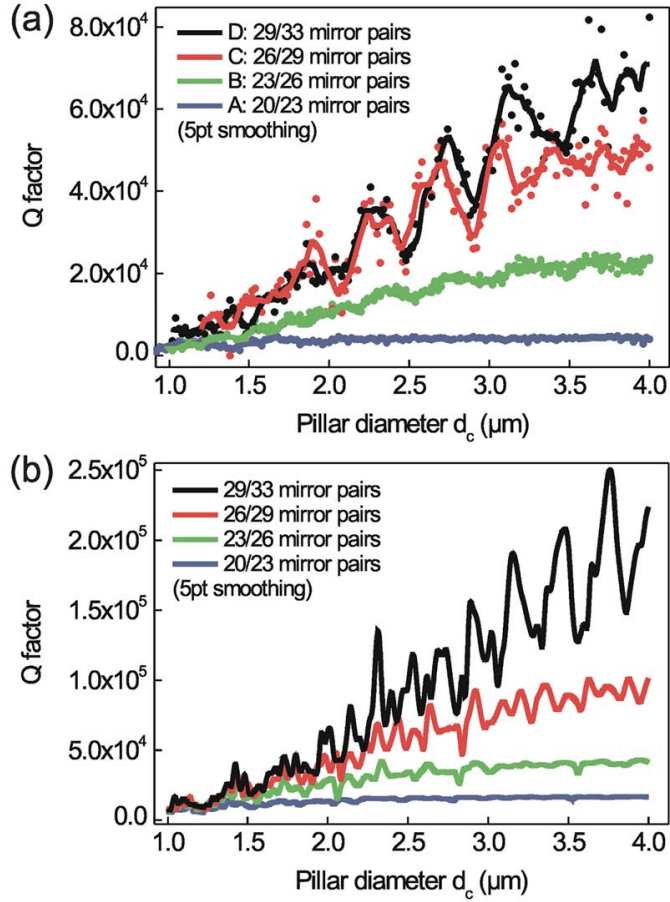

FIG. 3. (Color online) (a) Cavity $Q$ factors determined for samples A, B, C, and $\mathrm{D}$ with different numbers of mirror pairs in the DBRs (see legend) vs pillar diameter. (b) Calculated cavity $Q$ factors as a function of pillar diameter for DBR layouts corresponding to samples A-D.

to $23 / 26$, peaks and dips start to appear clearly. For $26 / 29$ pairs the oscillations are more pronounced, and for 29/33 periods corresponding to sample $\mathrm{D}$, we observe strong oscillations in the entire diameter range. It is interesting to note that the oscillatory behavior is caused by variations in the intrinsic $Q$ factor. This explains why the experimental $Q$ oscillations of sample D are slightly damped for large diameters when absorption losses become significant, while the amplitude of the oscillations continues to increase in the simulations which do not consider absorption losses.

The physical explanation for the appearance of strong oscillations for high- $Q$ micropillars in the long-diameter regime can be understood by examining the influence of the eigenvalue $R_{r}$ on the $Q$ factor. In Eq. (2) the derivative of the argument of $R_{r}$ is almost a constant function and the oscillations in $Q$ are contained in the first part $M$ given by

$$
M=\frac{1}{\left(1-\left|R_{r}\right|\right)} .
$$

When the micropillar diameter is extended, the number of bound higher-order modes is increased. Nonideal mode matching will result in coupling to higher-order modes when the fundamental mode is reflected by the DBRs. The interaction strength between the fundamental mode and the higher-order modes is weak, and for the low $Q$ micropillars examined in Refs. 7 and 8, the resulting perturbation to the eigenvalue $R_{r}$ is negligible compared to the value of $\left(1-\left|R_{r}\right|\right)$ for all but the first higher-order mode. This explains why a two-mode model is sufficient to predict the oscillatory behavior and why oscillations are absent for diameters above $\approx 1 \mu \mathrm{m}$ in Ref. 7. However, in our high $Q$ geometry, the denominator of Eq. (3) is two orders of magnitude smaller. Even though the interaction strength is unchanged, the per- turbations from modes of order beyond two are no longer negligible compared to $\left(1-\left|R_{r}\right|\right)$. Because the denominator of Eq. (3) is closer to zero, smaller variations in $R_{r}$ will influence the $Q$ factor resulting in distinct oscillations of $Q$ for diameters above $2.5 \mu \mathrm{m}$. This also explains why the oscillations become more pronounced as the number of DBR periods is increased. As the number of periods increases, $R_{r} \rightarrow 1$ and the sensitivity to perturbations from higher-order modes is enhanced.

Due to this non-negligible influence of higher-order mode coupling on $R_{r}$, a more advanced model than the twomode model in Refs. 7 and 8 is required to predict the positions of the peaks and dips in the oscillations of $Q$ in the large-diameter regime for high $Q$ geometries. However, we have not presented such a model since it is not within the scope of the present paper to find a fully quantitative agreement between experiment and simulation.

In conclusion we have demonstrated the existence of oscillatory behavior of $Q$ factors as a function of the diameter of high $Q$ micropillar cavities. The occurrence of the oscillations is related to a coupling of propagating Bloch modes at mirror interfaces. This mode coupling leads to variations in the DBR reflectivity when the pillar diameter is changed, a mechanism which is transferred into $Q$ oscillations in the medium diameter range $\left(1.0 \mu \mathrm{m}<d_{c}<4.0 \mu \mathrm{m}\right)$ in high $Q$ micropillars.

The authors acknowledge the fruitful discussion with J.-M. Gérard and P. Lalanne, and gratefully thank M. Emmerling and A. Wolf for expert sample preparation. This work was partially supported by the Deutsche Forschungsgemeinschaft via the Research Group "Quantum Optics in Semiconductor Nanostructures," the Danish Research Council for Technology and Production (Contract Nos. 274-070149 and 274-07-0230), the European Commission through the IST Project "QPhoton" (Contract No. IST-29283), and the State of Bavaria.

${ }^{1}$ K. J. Vahala, Nature (London) 424, 839 (2003).

${ }^{2}$ J. P. Reithmaier, G. Sẹk, A. Löffler, C. Hofmann, S. Kuhn, S. Reitzenstein, L. V. Keldysh, V. D. Kulakovskii, T. L. Reinecke, and A. Forchel, Nature (London) 432, 197 (2004).

${ }^{3}$ T. Rivera, J.-P. Debray, J. M. Gérard, B. Legrand, L. Manin-Ferlazzo, and J. L. Oudar, Appl. Phys. Lett. 74, 911 (1999).

${ }^{4}$ N. G. Stoltz, M. Rakher, S. Strauf, A. Badolato, D. D. Lofgreen, P. M. Petroff, L. A. Coldren, and D. Bouwmeester, Appl. Phys. Lett. 87, 031105 (2005).

${ }^{5}$ D. M. Whittaker, P. S. S. Guimaraes, D. Sanvitto, H. Vinck, S. Lam, A. Daraei, J. A. Timpson, A. M. Fox, M. S. Skolnick, Y.-L. D. Ho, J. G. Rarity, M. Hopkinson, and A. Tahraoui, Appl. Phys. Lett. 90, 161105 (2007).

${ }^{6}$ S. Reitzenstein, C. Hofmann, A. Gorbunov, M. Strauß, S. H. Kwon, C. Schneider, A. Löffler, S. Höfling, M. Kamp, and A. Forchel, Appl. Phys. Lett. 90, 251109 (2007).

${ }^{7}$ P. Lalanne, J. P. Hugonin, and J. M. Gérard, Appl. Phys. Lett. 84, 4726 (2004).

${ }^{8}$ G. Lecamp, P. Lalanne, J. P. Hugonin, and J.-M. Gérard, IEEE J. Quantum Electron. 41, 1323 (2005).

${ }^{9}$ G. Lecamp, J. P. Hugonin, P. Lalanne, R. Braive, S. Varoutsis, S. Laurent, A. Lemaâtre, and I. Sagne, Appl. Phys. Lett. 90, 091120 (2007).

${ }^{10}$ A. Löffler, J. P. Reithmaier, G. Sẹk, C. Hofmann, S. Reitzenstein, M. Kamp, and A. Forchel, Appl. Phys. Lett. 86, 111105 (2005).

${ }^{11}$ P. Bienstman and R. Beats, Opt. Quantum Electron. 33, 327 (2001).

${ }^{12} \mathrm{~N}$. Gregersen and J. Mork Opt. Quantum Electron. (in press).

${ }^{13}$ L. A. Coldren and S. W. Corzine, Spontaneous Emission and Laser Oscillation in Microcavities (CRC, Boca Raton, FL, 1995). 